\title{
Development of Electronic Cam Motion Control for Synchronous Cutting System
}

\author{
Adi Setiadi \\ Department of Mechanical Engineering \\ Swiss German University \\ Tangerang 15143, Indonesia \\ Email: adisetiadi31@gmail.com
}

\author{
Hanny J. Berchmans \\ Department of Mechanical Engineering \\ Swiss German University \\ Tangerang 15143, Indonesia
}

\begin{abstract}
Before the introduction of electronic motion controls, the traditional mechanical cam was the best approach to be used in applications that perform repetitive operations. Where the unique feature of the cam system is that the master-follower relationship is always repeated. When the electronic motion control system was introduced, the ability to duplicate the function of the mechanical cam becomes possible by electronic control drives called electronic cams. Limitation of speed, accuracy, and random space are some of the problems faced by mechanical cam. This study made a prototype of the high-speed rotary synchronous cutting system using electronic cam feature of motion controller Yaskawa MP-3000 Series with Sigma-7 servo system. This study has obtained and analyzed a model of the high-speed rotary cutting system, successfully controlled the rotary cutter motion and proved an alternative cam motion operation with electronic cam motion control. Performance and precision test for high-speed system need to be improved for the future study.
\end{abstract}

Keywords: Cams, Electronic Cam, Motion Control, Rotary Cutting, Development.

\section{INTRODUCTION}

Before the introduction of electronic motion controls, the traditional mechanical cam was the best approach to be used in applications that perform repetitive operations. In the cam system, the masterfollower relationship is always repeated, that is a unique feature of the cam system. A general camfollower system consists of three elements, that is a cam, follower, and driver. Cam is a rotating or oscillating object which provides reciprocating or oscillating motion to a second object, called a follower, which it touches (Fig.1). In general, a cam is mounted to a rotating motor shaft called the driver, with a constant velocity.

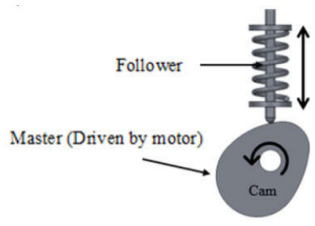

Fig. 1. Cam (master) - Follower Mechanism
When electronic motion control systems were introduced, the ability to duplicate mechanical cam functions became possible with electronically controlled drives called electronic cams. Electronic cam applications usually build a non-linear relationship between the master drive and follower drive where the cam input matches the position of the master drive and the cam output matches the followers drive position.

The synchronous cutting system (Fig.2) is a good example of application such as the requirement for a process involving the movement of continuous material, to cut a product to a certain length, it is important that the cutter must be synchronous with the movement of the material during cutting and between cutting operations. Compensation movements must be carried out between the cutting steps, according to the length of the product.

\section{OBJECTIVES}

The objective of this study is to conduct research in synchronous cutting systems by implementing the electronic cam system. The focus of this research is to replace the mechanical cam system and repetition operation of the electronic motion control system with electronic cam motion control systems. Specifically, the goal is to develop a prototype of cutting machines to analyze electronic cam motion control system capabilities and performance.

\section{Theoritical Perspectives}

\section{A. Mechanical Cam}

Cam is a rotating or oscillating object which provides reciprocating or oscillating motion to a second object, called a follower, which it touches (Fig.3). When the cam rotates, the followers are made rise, dwell, and fall; the length of time spent on a position depends on the shape of the cam. The rise cam part is a part that pushes the follower up, the profile determines how fast the cam follower will be lifted. The fall cam part is a part that which makes the follower lowered, the profile determines how fast the followers fall. The dwell cam part is where it rotates with an unchanging radius.

The cam shape needed to produce certain movements from followers. Figure 4 shows the type of follower displacement diagrams produced by eccentric cam with point-shaped followers. The figure shows how this radial distance, and 


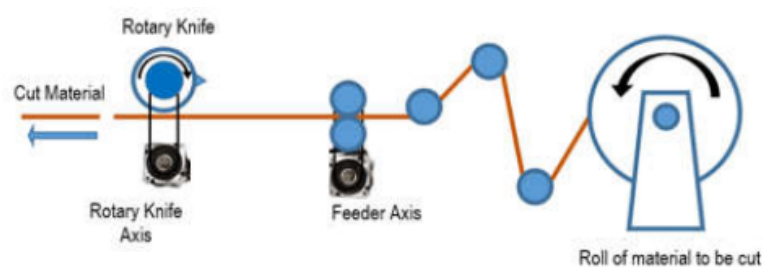

Fig. 2. A Synchronous Cutting System

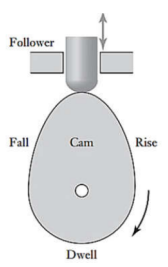

Fig. 3. Cam and Cam Follower

hence the displacement of followers, varies according to the rotation angle of the cam. The vertical displacement diagram is obtained by taking the radial distance of the cam surface from the rotation point at different angles and projecting it around to give displacement at that angle.

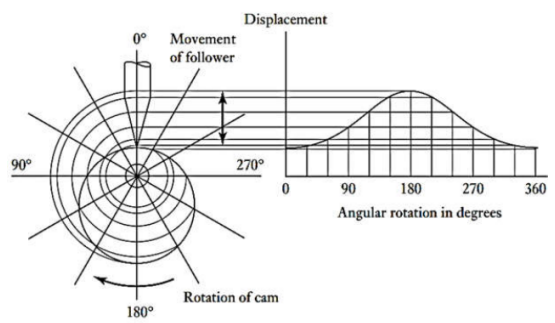

Fig. 4. This is a circular cam with a center of rotation offset. The results in follower oscillations that are simple harmonic motion. The radial distance from the axis of the cam rotation to the point of the cam contact with the follower gives the movement of followers by referring to the axis of rotation of the cam.

\section{B. Electronic Cam}

The electronic cam (Fig.5) control is a technique for achieving the operation of the machined cam by the motion control. The control that operates running on of the machine while completely keeping step with the movement of a certain standard is said, "Synchronous follow type control". There is one kind of electronic cam control about a synchronous follows type control. As for running one of the machines (slave), it is a pace to the movement of a certain standard (master) in complete. The control that operates while matching it is said. (synchronous follow type control).

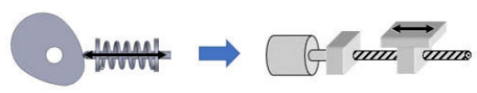

Fig. 5. Electronic cam achieving operation of the machined cam.

In general, electronic camming is accomplished with servomotors, actuators and drives and controllers with electronic gearing functions. With electronic cam, the mechanical setup is replaced by electronics, that is motion profile function. These cam profiles are typically defined in a table as a set of $x-y$ points.
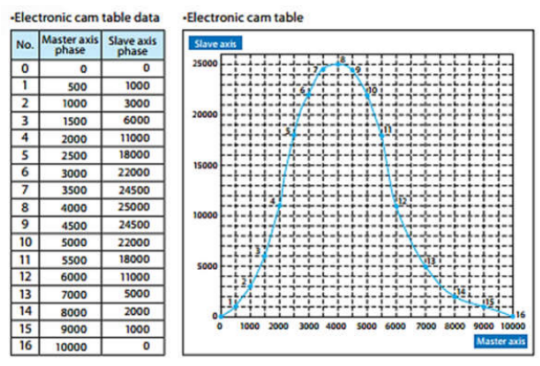

Fig. 6. Cam profiles generate from electronic cam table data.

\section{Motion Control Servo System}

Elements of a servo system include motors, encoders, drivers, and controllers. According to the Japanese Industrial Standard (JIS) terminology, a "Servo-mechanism" is a mechanism that uses the position, direction or orientation of an object as a variable of the process in a control system to follow any change in target value (setpoint). (Source: JIS B0181). 


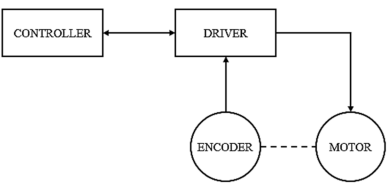

Fig. 7. Cam profiles generate from electronic cam table data.

Servo systems can be defined in more detail as a mechanism that moves at a specified speed and locates an object in a specified position. Therefore, in developing a servo system should be designed an automatic control system equipped with feedback control (closed-loop control). Figure 8 below shows the configuration of motion control system using servo system.

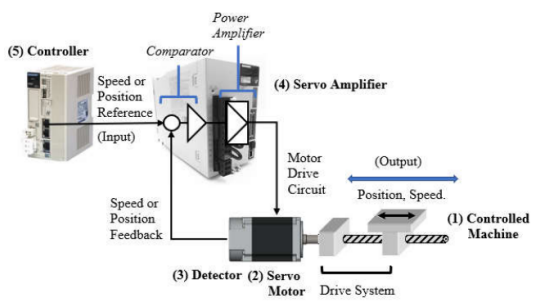

Fig. 8. Configuration of a motion control system using servo system.

1) Controlled machine: Mechanical system whose position or speed must be controlled. This includes a drive system that transmits torque from the servomotor.

2) Servomotor: The main actuator that drives a controlled system. There are two types: servomotor AC and DC servomotor.

3) Detector: Detector position or speed. Usually, an encoder installed on a motor is used as a position detector.

4) Servo Amplifiers: Amplifiers that process error signals to correct differences between reference and feedback data and operate the appropriate servomotor. The servo amplifier consists of a comparator, which processes errors signal, and power amplifier, which operates the servomotor.

5) Controller: A device that controls the servo amplifier by determining position or speed as a set point.

\section{DESIGN OF EXPERIMENT}

\section{A. Prototype Cutting Machine Design}

Prototype of rotary cutting system design consists of conveyor belt design and rotary cutter design. Design of the rotary cutting system (Fig.9).

The leftmost pulley is a drive pulley, describe as $\mathrm{P} 1$ is driven by a servo motor which will allow velocity $(\omega \mathrm{P} 1)$ feedback and control. The other pulleys which are described as P2 are

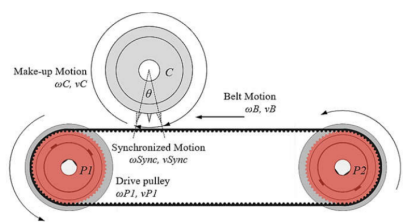

Fig. 9. Prototype of rotary cutting system design.

idle rollers. When the conveyor belt correctly tensioned, they have no slippage and then the timing belt and pulley will be synchronous. That mean need to install pulley of conveyor properly with the correct centre to centre pulley distance which should be calculated.

$$
\omega_{P 1}=\omega_{B e l t}, v_{P 1}=v_{B e l t}
$$

where:

$\omega_{P 1}, v_{P 1}=$ Velocity of the drive pulley and $\omega_{\text {Belt }}, v_{\text {Belt }}=$ Velocity of the timing belt.

There come two types of motion zones of the rotary cutter, which is synchronous motion (Sync) when the cutting process occurs, and the make-up motion (Make-up) before the cutting process. For linear velocity of synchronous motion $\left(v_{S y n c}\right)$ must same with linear velocity of the conveyor belt to avoid tension and deformation on the web/sheet material when the cutting process.

$$
v_{\text {Sync }}=v_{\text {Belt }}
$$

where: $v_{\text {Sync }}=$ Velocity of the cutter synchronous motion.

For linear velocity of make-up motion $\left(v_{\text {Makeup }}\right)$ has three case variations depending on the length of the web/sheet material to be cut compared to the circumference of the cutter.

TABLE I

LINEAR VELOCITY VARIATION OF CUTTER MAKE-UP MOTION

\begin{tabular}{cll}
\hline Case & Condition & Remarks \\
\hline 1 & $\begin{array}{l}L_{\text {Cut }}=C_{\text {Cutter }} \\
v_{\text {Makeup }}=v_{\text {Belt }}\end{array}$ & $\begin{array}{l}\text { Linear velocity of cutter make-up motion } \\
\text { will be same with the conveyor belt linear } \\
\text { velocity }\end{array}$ \\
2 & $\begin{array}{l}L_{\text {Cut }}>C_{\text {Cutter }} \\
v_{\text {Makeup }} \\
v_{\text {Belt }}\end{array}$ & $\begin{array}{l}\text { Linear velocity of cutter of make-up motion } \\
\text { should be slower than the conveyor belt } \\
\text { speed to provide more time on the web/sheet } \\
\text { material to pass the rotary cutter position } \\
\text { and longer material cutting. }\end{array}$ \\
& $\begin{array}{l}L_{\text {Cut }}<C_{\text {Cutter }} \\
v_{\text {Makeup }} \\
v_{\text {Belt }}\end{array}$ & $\begin{array}{l}\text { Linear velocity of cutter make-up motion } \\
\text { should be faster than the conveyor belt } \\
\text { speed to provide short time on the web/sheet } \\
\text { material to pass the rotary cutter position } \\
\text { and shorter material cutting. }\end{array}$ \\
\hline
\end{tabular}

Geometrical design of the conveyor system shown at Figure 10. The belt used is Powerdrive 610-2GT-06 GT2 timing belt with total pitch length is $610 \mathrm{~mm}$. And then, the pulley used is Powerdrive 60-2MGT-06-6FA timing pulley with a number of teeth is 60 teeth. For simple drives consisting of pulleys 


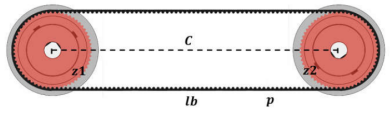

Fig. 10. Geometrical design of conveyor.

with an equal number of teeth (ratio $i=1$ ) the centre distance can be found from:

$$
C=\frac{p}{2}\left(z_{b}-z\right)=\frac{1}{2}\left(l_{b}-p . z\right)
$$

where: $C=$ Centre distance $(\mathrm{mm}), l_{b}=$ Belt length $(\mathrm{mm})$, $p=$ Tooth pitch (mm), $z=$ Number of teeth in the pulley, $z_{b}$ $=$ Number of teeth in the belt.

$$
\begin{aligned}
C=\frac{1}{2}\left(l_{b}-p . z\right) & =\frac{1}{2}(610-(2 \times 60)) \\
& =245 \mathrm{~mm}
\end{aligned}
$$

The cutter is mounted on a circular body which has a diameter $43 \mathrm{~mm}$ (Fig.11). Then the circumference of the circle from the rotary cutter body needs to be calculated to determine the speed of the rotary cutter later.

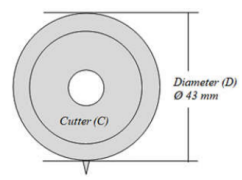

Fig. 11. Rotary cutter design.

Calculation of circumference rotary cutter.

$$
\begin{aligned}
C_{\text {Cutter }}=\pi \times D & =3.14 \times 43 \\
& =135 \mathrm{~mm}
\end{aligned}
$$

The speed of the conveyor belt can be expressed as:

$$
\begin{aligned}
v_{\text {Belt }}=\frac{\pi \times D \times n m}{60} & =\frac{\pi \times 0.043 \times 3000}{60} \\
& =6.751 \mathrm{~m} / \mathrm{s}
\end{aligned}
$$

where: $v_{\text {Belt }}=$ Velocity of belt $(\mathrm{m} / \mathrm{s}), D=$ Diameter of pulley $(\mathrm{m}), n m=$ Speed of servomotor $(\mathrm{rpm})$.

\section{B. System Control Design}

In Figure 13, the system control configuration for the prototype of rotary cutting system that consists of 1 unit Yaskawa MP3000 motion controller with IO, 2 unit Yaskawa Sigma-7 servo amplifier and servo motor for axis cutter and axis conveyor, and 1 unit photoelectric sensor for detect the material. Communication between the controller and amplifier are using mechatrolink system (fieldbus).

Programming approach to control the cutting machine is the controller set the speed control command to the master axis

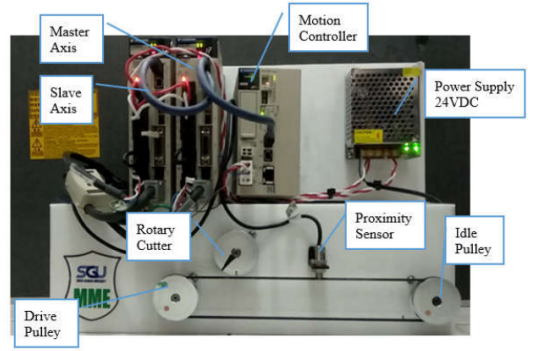

Fig. 12. Prototype of rotary cutting system.

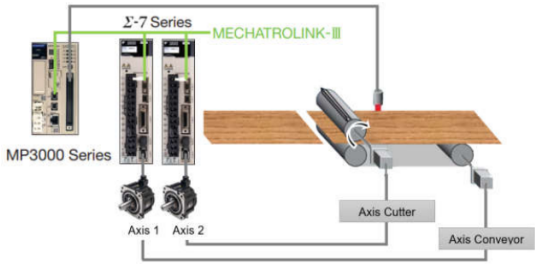

Fig. 13. System control configuration.

and the feedback position of the master axis use as reference phase control command to the slave axis based on electronic cam profile which is generated from the electronic cam table data.

\section{RESULT OF EXPERIMENT}

The experiment result has been done with three different case of cutter motion velocity, that can be same, faster, or slower than the velocity of belt. Because of that, need to test the precision of the system at those three different cases. The speed of master axis for precission test is assumed to be constant at $500 \mathrm{rpm}$. The precision test method is a visual check cutting position and analysis graph of the coordinate feedback from the encoder signal of each axis.

A. Precission test Case 1: Length material to be cut = circumference of cutter $\rightarrow 135 \mathrm{~mm}$

The graph on the Figure 14 shows that the cutter keeps moving, slowing down between cuts. In the case where the cut length of the material equal to the rotary cutter body circumference.

B. Precission test Case 2: Length material to be cut ¿ circumference of cutter $\rightarrow 270 \mathrm{~mm}$

The graph on the Figure 15 show that the cutter stops at home position between cuts, waiting for another cut point. In the case where the cut length is chosen longer than the rotary cutter circumference. 


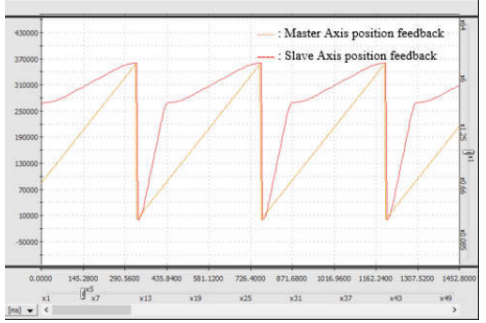

Fig. 14. Graph of coordinate feedback position case-1

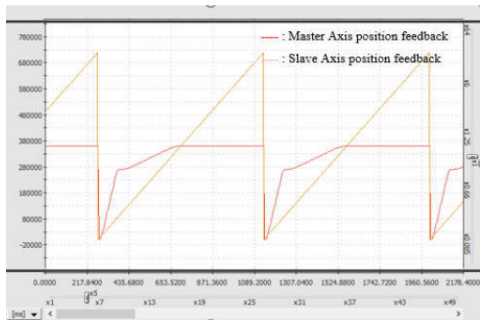

Fig. 15. Graph of coordinate feedback position case-2.

\section{Precission test Case 3: Length material to be cut ; circum- ference of cutter $\rightarrow 67,5 \mathrm{~mm}$}

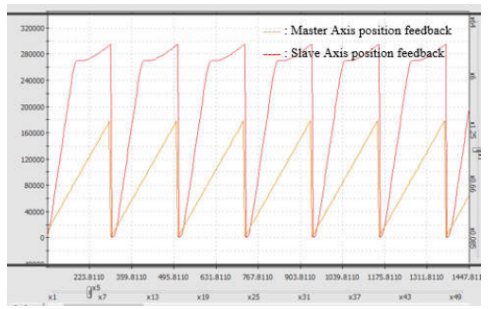

Fig. 16. Graph of coordinate feedback position case-3.

The graph on the Figure 16 show that the cutter keeps moving, slowing down between cuts. In the case where the cut length of the material equal to the rotary cutter body circumference.

\section{CONCLUSION}

This study has obtained and analized a model of the high-speed rotary cutting system, successfully controlled the rotary cutter motion and proved an alternative cam motion operation. Here is some advantage when applied electronic cam. Electronic cam has the following features compared with the mechanical cam.

1) The structure of mechanical cams is complicated and when need to change the motion, need to change the cams. Changing the cams takes a long time. However, for electronic cam, only need to load new cam data to the controller.

2) For mechanical cam, producing the cam itself is a hard job, but also, getting high accuracy is not easy. But for electronic cam, because of digital data, the accuracy of cam motion is not affected by the production of the cam itself.

3) For mechanical cam, followers movements always depend on the master position. But for electronic cam, some time can pass through the master position, then random feeds can be easily controlled.

4) When mechanical cam creates the motion of a big stroke, the link structure is used and this amplifies the error of cams. However for the electronic cam, there is an only error of a ball screw, and this error is known from the selection of the screw. There is no amplification of errors.

5) For mechanical cam, when need to increase the speed, step parts of the cam will be skipped. This is because the speed is too fast to follow every part of the surface of the cam. However, for electronic cam, a ball screw and a servo motor are only used to imitate the motion and there is no skipping.

6) For mechanical cam, cams need to be maintained and managed in storage and remember the motion itself but for electronic cam, the data is stored in computer memory and easy to simulate.

\section{RECOMMENDATION}

This study can be applied in application high-speed camming systems to obtain more efficient and smooth motion operations. This improvement could save costs on reducing waste product and also can realize high speed and high accuracy system. In addition, for future works, about performance and precision test for the high-speed system can use a highspeed camera to see if the motion are accurate and make some analysis about the mechanical part.

\section{REFERENCES}

[1] George W. Younkin, 2003. Industrial Servo Control Systems: Fundamentals and Applications. Industrial Controls Consulting, Inc. Fond du Lac, Wisconsin, U.S.A.

[2] Mike Woelfel. 1999. Introduction to electronic cam. Assembly Automation. 19(1): p. 17-24

[3] Raimund Perneder. 2012. Handbook Timing Belts: Principles, Calculations, Applications. Berlin,Germany: Springer-Verlag Berlin Heidelberg.

[4] Riazollah Firoozian. 2014. Servo Motors and Industrial Control Theory. Springer. Switzerland.

[5] William Bolton. 2015. Mechatronics: Electronic control systems in mechanical and Electrical Engineering. Pearson.

[6] Yaskawa. 2014. Machine Controller MP2000/MP3000 Series Setup Manual. Yaskawa Electric Corporation, Japan

[7] Yaskawa. 2004. Machine Controller MP900/MP2000 Series Operation Manual : Electronic cam data Preparation tool. Yaskawa Electric Corporation, Japan. 